\title{
Feasibility of combining gait robot and multichannel functional electrical stimulation with intramuscular electrodes
}

\author{
Jessica P. McCabe, MPT; ${ }^{*}$ Mark E. Dohring, PhD; ${ }^{1}$ E. Byron Marsolais, MD, PhD; ${ }^{1-2}$ Jean Rogers, PT; $^{1}$ \\ Richard Burdsall, PT; ${ }^{1}$ Kristen Roenigk, BME; ${ }^{1}$ Svetlana Pundik, MD; ${ }^{1,3}$ Janis J. Daly, PhD, MS ${ }^{1,3}$ \\ ${ }^{1}$ Cognitive and Motor Learning Laboratory, Cleveland FES Center, Louis Stokes Cleveland Department \\ of Veterans Affairs Medical Center, Cleveland, $\mathrm{OH} ;{ }^{2}$ Departments of Anesthesiology, Biomedical Engineering, and \\ Orthopaedic Surgery, and ${ }^{3}$ Department of Neurology, Case Western Reserve University School of Medicine, Cleveland, $\mathrm{OH}$
}

\begin{abstract}
After stroke rehabilitation, many survivors of stroke exhibit persistent gait deficits. In previous work, we demonstrated significant gains in gait kinematics for survivors of chronic stroke using multichannel functional electrical stimulation with intramuscular electrodes (FES-IM). For this study, we tested the feasibility of combining FES-IM and gait robot technologies for treating persistent gait deficits after stroke. Six subjects, $>/=6$ months after stroke, received 30-minute intervention sessions of combined FES-IM and gait robotics 4 days a week for 12 weeks. Feasibility was assessed according to three factors: (1) performance of the interface of the two technologies during intervention sessions, (2) clinicians' success in using two technologies simultaneously, and (3) subject satisfaction. FES-IM system hardware and software design features combined with the gait robot technology proved feasible to use. Each technology alone provided unique advantages and disadvantages of gait practice characteristics. Because of the unique advantages and disadvantages of each technology, gait deficits need to be accurately identified and a judicious treatment plan properly targeted before FES-IM, a gait robot, or both combined are selected.
\end{abstract}

Clinical Trial Registration: Feasibility and Practice Characteristics of FNS and Gait Robot; NCT00508755; Study ID: B4036I, <www.clinicaltrials.gov>.

Key words: cerebrovascular accident, FES, functional electrical stimulation, gait, gait robot, gait training, hemiplegia, motor learning, rehabilitation, robotics, stroke.

\section{INTRODUCTION}

After stroke rehabilitation, many survivors of stroke still exhibit persistent gait deficits. In previous work, we have demonstrated significant gains in gait kinematics for treating survivors of chronic stroke using functional electrical stimulation (FES) with intramuscular electrodes (FES-IM) [1-2]. FES-IM gait training provided subjects with a practice pattern that approximated normal movement of the hip, knee, and ankle during the swing phase of gait. It also provided subjects with the ability to practice knee control during the stance phase of gait. A disadvantage of FES-IM alone was that more than 6 months was required to produce results in some cases. Therefore, we successfully combined FES-IM and bodyweight-supported (BWS) treadmill training (BWSTT)

Abbreviations: BWS = body-weight-supported, BWSTT = BWS treadmill training, FES = functional electrical stimulation, FES-IM = FES with intramuscular electrodes, GAIT = Gait Assessment and Intervention Tool, GF = guidance force, $\mathrm{IM}=$ intramuscular, SCI = spinal cord injury, VA = Department of Veterans Affairs.

*Address all correspondence to Jessica P. McCabe, MPT; Cognitive and Motor Learning Laboratory, Louis Stokes Cleveland VA Medical Center, 10701 East Blvd, 151-W, Cleveland, OH 44106; 216-791-3800, ext 4691; fax: 216-2298509. Email: JessicaPMcCabe@hotmail.com

DOI: 10.1682/JRRD.2007.08.0124 
[3]. Combined use of FES-IM and BWSTT provided gait practice of more of the desired coordinated gait components than did either modality alone [4]. In fact, the combination FES-IM and BWSTT produced significant gains in gait components and knee-flexion coordination versus BSWTT alone [3,5-6]. However, not all subjects demonstrated gait restoration to normal (i.e., normal coordinated gait components) [7].

A shortcoming of the combined intervention of FESIM and BWSTT was that early in the gait training practice, close-to-normal swing-phase movements were not always possible to provide. And though BWSTT alone provided stance-phase practice characteristics [8-10], it did not assist with swing-phase movement for gait practice [4]. FES-IM did provide close-to-normal swingphase movements for gait practice [1-2], but for some patients, this close-to-normal practice was possible only later in the gait training protocol, after some musclestrength restoration and joint-movement coordination.

Gait robots were developed to assist in gait training. One randomized controlled trial of gait robotics illustrated some potential in patients who had a stroke. That trial showed that an advantage of gait robot training versus conventional gait training existed in terms of singlelimb stance duration [11]. This finding was promising, but no significant group difference was found for any other measure [11]. One gait robot [12] (Lokomat, Hocoma Inc; Chicago, Illinois) could provide assisted or passively induced swing-phase gait movements that were close to normal early after injury or stroke. One disadvantage of a gait robot is that passive movement practice is not optimal for motor learning. However, in contrast to robotics, electrically induced muscle activation can provide practice of active joint movement. Given the disadvantages of each technology alone and the potential advantages of the combination, this study assessed the feasibility of combining FES-IM and a gait robot for treating persistent gait deficits after stroke. The hypothesis was that combining FES-IM and a gait robot will be feasible for gait training in patients who had a stroke. The objectives included (1) training clinicians in the delivery of each technology alone and the two technologies simultaneously and (2) identifying which coordinated gait components could be practiced with each technology alone and with the two technologies combined.

\section{METHODS}

\section{Subjects}

We enrolled six subjects who had had a stroke $\geq 6$ months before the study. Inclusion criteria were sufficient endurance to participate in rehabilitation sessions; ability to follow two-stage commands; medically stable; an equal or greater than trace contraction of hip, knee, and ankle flexors and extensors [13]; and Fugl-Meyer lowerlimb muscle motor score of $\geq 32$ points. Additional inclusion criteria were inability to flex the knee and ankle in the sagittal plane in a normal manner during walking so the limb cleared the floor and inability to control normal knee angle during single-limb weight-bearing stance phase. Subjects provided informed consent according to the Declaration of Helsinki, and the Louis Stokes Department of Veterans Affairs (VA) Medical Center Institutional Review Board (committee on human subjects' protection in research) approved the study.

\section{Equipment and Technology}

\section{Gait Robot}

We used the Lokomat gait robot system, consisting of a robotic-driven orthosis, a BWS system, and a treadmill (Woodway; Rhein, Germany). We used gait speeds that ranged from 1.0 to $2.9 \mathrm{~km} / \mathrm{h}$. The robotic-driven orthosis provided the subjects sagittal plane and hip and knee joint movement that was adjusted to optimize gait kinematics at the hip and knee joints and restricted to a physiologically safe range. The ankle was controlled passively by a dorsiflexion spring-loaded stirrup.

\section{FES-IM}

The FES-IM system consisted of eight intramuscular (IM) electrodes, an external stimulator worn on a belt, and a finger switch. Electrodes were placed at the motor point of up to eight paretic or paralyzed muscles: tibialis anterior, peroneus longus, lateral head of the gastrocnemius, short head of the biceps femoris, semitendinosus, semimembranosus, vastus lateralis, and gluteus medius. Detailed descriptions are available in prior publications for the electrode technology [14], the electrode placement procedure [15], and electrode performance for patients after stroke [16]. The stimulator was used to control the eight IM electrodes [17]. Template patterns were individualized to practice FES-assisted movements and gait components [1]. 
FES-IM patterns were created with a specialized computer program [17]. The computer program provided the capability for therapists to develop a stimulation pattern with up to eight muscles in multiple combinations and timing variations. The individualized FES-IM patterns were downloaded to the subject's stimulator for gait training. The therapist or subject controlled the stimulator by accessing a menu using a finger switch. For the stimulus, the parameters used were $33 \mathrm{~Hz}$ frequency, $20 \mathrm{~mA}$ amplitude, and 4 to $150 \mu$ s pulse width. First, comfortable pulse-width range was ascertained, followed by determination of the desired muscle response within the subject's comfort level. Then the timing of muscle contraction within the stimulation pattern was programmed by the therapists to obtain movement as close to normal as possible.

\section{Treatment Protocol}

\section{Decision-Making Criteria for Treatment Initiation and Progression}

Subjects received 30-minute intervention sessions of combined FES-IM and gait robot 4 days a week for 12 weeks. We selected the Gait Assessment and Intervention Tool (GAIT) to assess gait deficits and the need for robotics and/or FES-IM assistance. The GAIT is a comprehensive, visual gait analysis measure with good interand intrarater reliability [18] that scores coordinated movements composing each phase of the gait cycle (World Congress of Physical Therapy, http://www.wcpt.org/ abstracts2007/WCPT2007.html) [18]. We relied on the GAIT item scores to determine the aspects of treatment regarding optimal training parameters during gait robot training and stimulation pattern development. In addition, we identified the subject's chosen overground gait speed and training speed/percent of BWS during conventional BWSTT to guide the initial gait robot training speed and level of BWS in the gait robot.

\section{FES-IM Gait Training}

From preexisting template FES-IM patterns, we developed customized FES-IM patterns to address both swing-phase and stance-phase gait deficits for each subject. After a custom FES-IM pattern was developed, the pattern was tested while subjects walked in the parallel bars. We iteratively modified the FES-IM pattern to create an optimal pattern to practice components of gait that were as close to normal as possible. The performance of both the subject and the FES-IM pattern was evaluated during each intervention session, and the FES-IM assistance was reduced over time so that greater volitional control was demanded as volitional control improved.

\section{Preparing for Gait Robot Training}

During the first gait robotics training session, we iteratively modified the following parameters to optimize the gait pattern delivered by the gait robot: limb joint range of motion at the hip and knee, treadmill speed, level of BWS, and level of assistance or guidance force (GF) provided by the gait robot. Initial training speed was derived from the subject's chosen overground speed. We obtained initial percent of BWS during conventional BWSTT to establish initial levels of BWS during training with the gait robot. Training intensity was monitored according to heart rate and blood pressure, as well as subject's report of his or her rating of perceived exertion during training.

\section{Combining FES-IM and Robot Gait Training}

At the initial intervention session, the subject trained in the gait robot without FES-IM. The subject's gait cycle swing-phase and stance-phase durations were timed with a stopwatch. In addition, gait analysis was performed during use of the gait robot. From these data, customized FES-IM stimulation patterns for stance phase and swing phase were devised and tested for comfort and performance while subjects walked in the parallel bars. At the second training session, the combined use of the gait robot and the new FES-IM patterns was tested. In subsequent intervention sessions, the combined use of the FES-IM and gait robot was analyzed and modifications were made so that greater volitional control was demanded as subjects regained volition control of portions of the gait pattern.

If the gait pattern performance was close to normal and the subject reported a low level of exertion, then the clinician reduced the FES-IM and gait robotics assistance to minimize technology assistance and demand greater volitional gait component performance, reducing BWS, increasing speed, reducing GF, and reducing FES-IM assistance, in that order.

\section{Outcome Measures}

We assessed feasibility of combining FES-IM and gait robot technologies according to three factors: (1) performance of the interface of the two technologies during intervention sessions, (2) clinicians' success in using the two modalities simultaneously, and (3) subject satisfaction. 
Performance of Interface Between FES-IM and Gait Robot Technologies

We assessed the performance of the interface between FES-IM and the gait robot systems in three ways. First, we assessed the interface between the subject FES-IM percutaneous lead wires and gait robot device. The FES-IM lead wire exit site integrity was assessed according to the maintenance of a clear space for the electrode lead wires on the anterior thigh, with no interference from the gait robot thigh cuff. The gait robot thigh-cuff position was monitored for any evidence of shifting during training.

Second, we assessed the FES-IM device cables and gait robot interface according to the feasibility of maintaining a clear gait training work space during the use of the gait robot and FES-IM systems. The FES-IM external wires and/or cables included the subject's percutaneous lead wires, the external connector, the stimulator cable, the ground cable, and the finger-switch cable. Cable malfunction was monitored during each intervention session.

Third, we assessed the simultaneous control of the FES-IM and the gait robot systems. We assessed the clinician's ability to safely and accurately control the gait robot while activating the stimulator finger switch for FES-IM system activation at the correct time in the gait cycle.

\section{Clinician Skill Factors Required for Delivery of Combined FES-IM and Gait Robot}

We evaluated the feasibility of clinicians successfully delivering the combined FES-IM and gait robot treatment. Three clinicians were evaluated for their ability to individualize the gait robot settings for their subjects, develop customized FES-IM patterns to address swingand stance-phase gait deficits, and combine the FES-IM and gait robot during intervention sessions.

The clinicians received training in using the gait robot and computer software for FES-IM patterns. The clinicians were then tested on seven skill factors:

1. Ability to adjust gait robot for subject training.

2. Accurate placement of FES-IM external cables/stimulator to prevent disconnection.
3. Correct placement of FES-IM pin connector on subject's thigh to maintain FES-IM lead wire exit site skin integrity.

4. Ability to customize FES-IM swing-phase pattern.

5. Ability to customize FES-IM stance-phase pattern.

6. Simultaneous accurate timing of FES-IM swing-phase activation with gait robot: toe-off to heel strike (beginning and end of swing phase).

7. Simultaneous accurate timing of FES-IM stance-phase activation with gait robot: heel strike to toe-off (beginning and end of stance phase).

\section{Subject Satisfaction}

A written questionnaire was used to query subjects regarding inconveniences, pain, or discomfort associated with the FES-IM and/or gait robot systems, malfunction of the FES-IM system, and overall satisfaction level with using the combined technologies.

\section{RESULTS}

\section{Subjects' Characteristics}

Six subjects were enrolled, aged 47 to 77 , who were 0.5 to $1.5 \mathrm{yr}$ after stroke (Table 1). Stance-phase gait deficits included an abnormally reduced weight shift to the involved limb and poor control at the hip, knee, and ankle. Swing-phase deficits included reduced hip, knee, and ankle flexion.

\section{Feasibility of Combined FES-IM and Gait Robot}

\section{Performance of Interface Between FES-IM and Gait} Robot Technologies

Table 2 provides results regarding performance of the three technology interface factors that were relevant during combined use of FES-IM and the gait robot: (1) lead wire exit sites, (2) cables, and (3) stimulator and finger switch. Overall, the hardware and software design features of the FES-IM system proved feasible to use in combination with the gait robot technology.

Table 1.

Subject characteristics.

\begin{tabular}{|c|c|c|c|c|c|c|c|c|}
\hline \multirow{2}{*}{$\begin{array}{c}\text { Total } \\
\text { Subjects }\end{array}$} & \multicolumn{2}{|c|}{ Stroke Type } & \multicolumn{2}{|c|}{ Stroke Location } & \multicolumn{2}{|c|}{ Poststroke (yr) } & \multicolumn{2}{|c|}{ Age Range (yr) } \\
\hline & Ischemic & Hemorrhagic & Cortical & Subcortical & $0.5-1.0$ & $>1.0$ & $45-59$ & $\geq 60$ \\
\hline 6 & 6 & 0 & 4 & 2 & 2 & 4 & 2 & 4 \\
\hline
\end{tabular}


Table 2.

Combined use of three interface factors functional electrical stimulation with intramuscular electrodes (FES-IM) and gait robot technology (lead wire exit sites, cables, and stimulator and finger switch) for subjects (S) 1-6 after stroke.

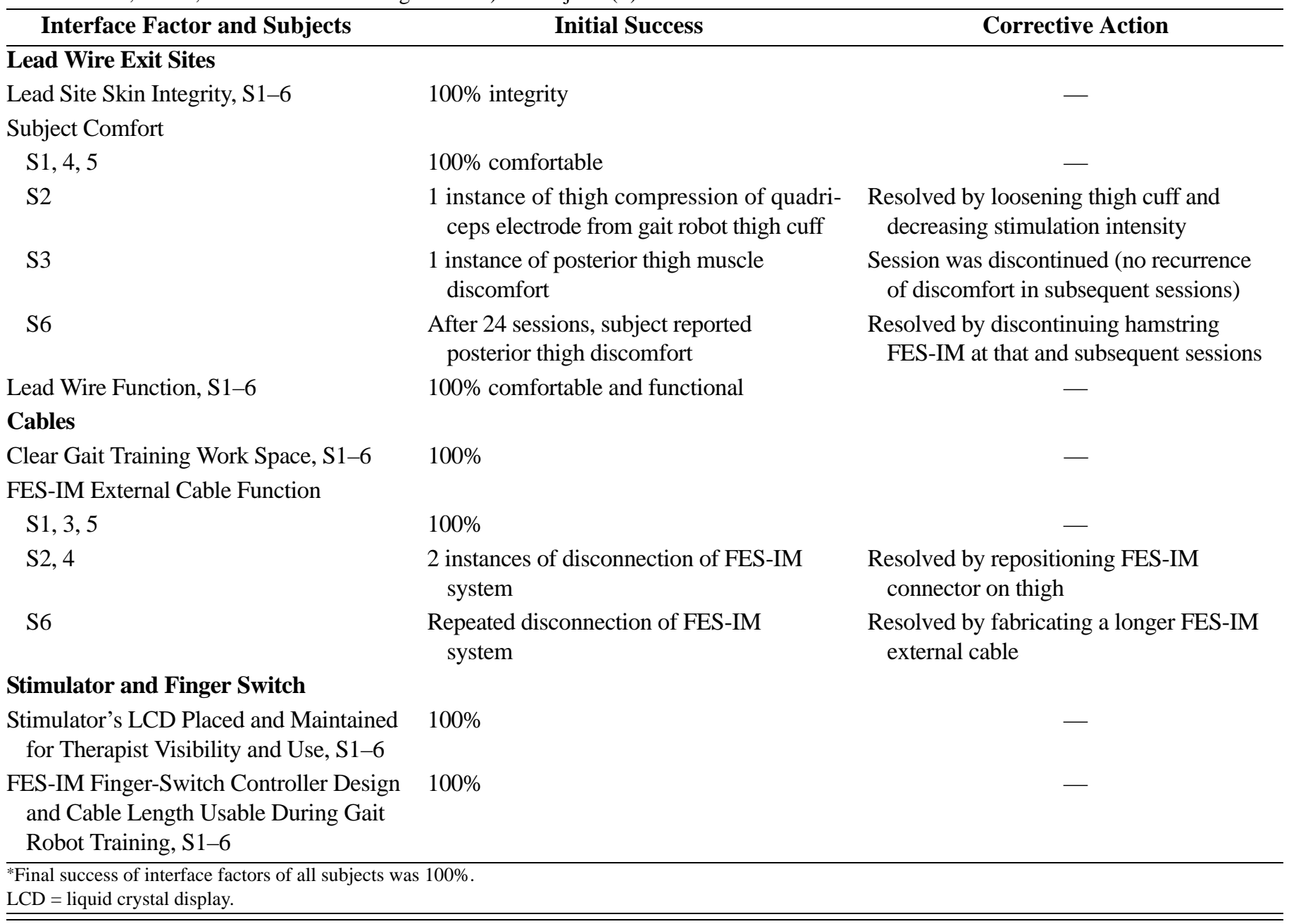

\section{FES-IM Pattern Delivery and Gait Robot Training}

Delivery of separate FES-IM patterns proved feasible for either stance or swing phase of gait simultaneously with gait robot operation. We developed a protocol to prepare for and provide gait practice with simultaneous assistance from both the FES-IM and gait robot systems.

\section{Protocol Developed for Simultaneous Provision of FES- IM and Gait Robot}

We developed and tested the following protocol for providing simultaneous FES-IM and gait robot training:

- Customize FES-IM swing-phase pattern for a given subject during overground walking.

- Test FES-IM swing-phase pattern using treadmill and BWS.
- Test FES-IM swing-phase pattern during "air walking," using 100 percent BWS and gait robot GF for the lower limbs.

- Select initial gait robot speed according to subject report of preferred overground speed.

- Refine FES-IM swing-phase pattern timing for use with gait robot using gait event timings obtained during subject walking in the gait robot at preferred speed (without FES-IM).

This protocol would also include practice of manual synchronization of FES-IM and gait robot swing phase without the subject according to simultaneous visual monitoring of the gait robot at desired speed as well as the following:

- FES-IM pattern onset and gait robot toe-off.

- FES-IM pattern deactivation and gait robot heel strike. 
Simultaneously providing FES-IM and gait robot training proved feasible, since the FES-IM stimulator liquid crystal display could be physically placed near the treadmill at the location of toe-off. In this way, both FESIM pattern timing and gait robot events could be visually monitored simultaneously. This protocol was also followed to develop and deliver FES-IM stance-phase patterns for simultaneous use with the gait robot.

\section{Feasibility of Clinician Delivery of Combined FES-IM and Gait Robot}

Clinician performance for three clinicians was assessed for the seven skill factors listed in Figure 1. Within three sessions, clinicians achieved 7/7 skill factors.

\section{Subject Response and Satisfaction}

According to the satisfaction survey, two subjects reported no discomfort with use of either of the modalities or when the technologies were combined. One subject reported several instances of mild discomfort when the FES-IM system activated the knee flexors. One subject reported an isolated incident of mild discomfort on the lateral aspect of the calf during combined FES-IM and gait robot training. In all cases, discomfort was alleviated with the decrease of stimulation intensity. Table 3 provides data before and after treatment for the 6-minute walk test.

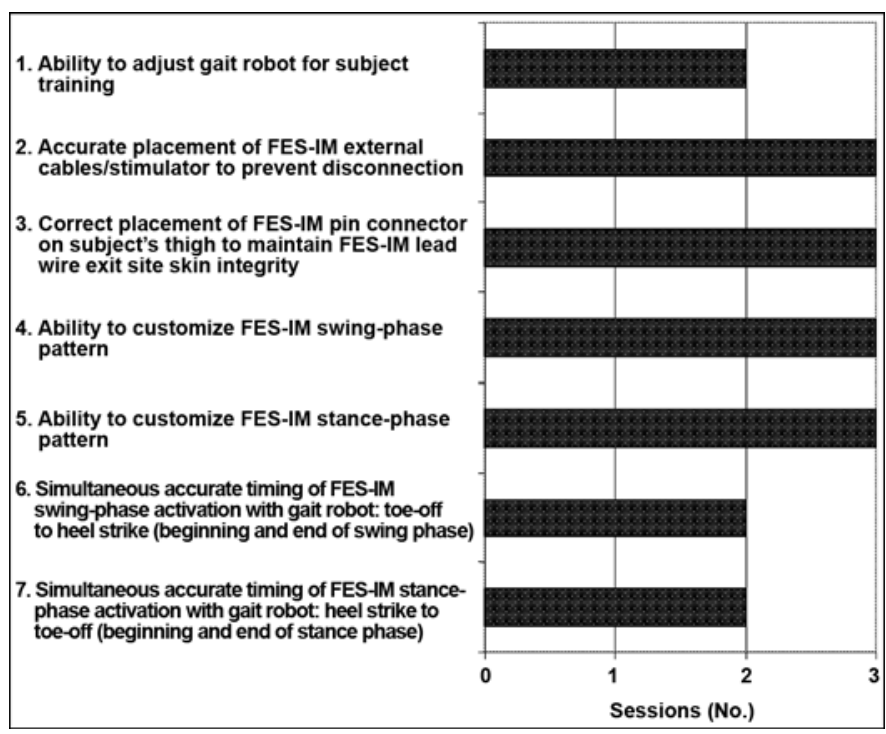

Figure 1.

Within three training sessions, clinician skill factors were mastered for delivery of combined functional electrical stimulation with intramuscular electrodes (FES-IM) and gait robot systems.
Table 3.

Initial and posttreatment scores for 6-minute walk test of six subjects after stroke.

\begin{tabular}{cccc}
\hline $\begin{array}{c}\text { Subject } \\
\text { No. }\end{array}$ & $\begin{array}{c}\text { Initial Score } \\
\text { (ft) }\end{array}$ & $\begin{array}{c}\text { Postscore } \\
\text { (ft) }\end{array}$ & $\begin{array}{c}\text { Change } \\
\text { (ft) }\end{array}$ \\
\hline 1 & 374 & 426 & 52 \\
2 & 307 & 455 & 148 \\
3 & 774 & 707 & $-67^{*}$ \\
4 & 548 & 678 & 130 \\
5 & 794 & 602 & $-192^{\dagger}$ \\
6 & 634 & 714 & 80 \\
\hline
\end{tabular}

*Subject had onset of two seizure episodes, subsequently controlled by medication under supervision of primary care physician. Subject completed study.

${ }^{\dagger}$ Subject developed Baker's cyst after enrollment and was discontinued from study. Subject completed 8/48 sessions ( $17 \%$ of total protocol visits).

\section{Comparison of Gait Practice Characteristics of FES-IM and Gait Robot}

Tables 4 provides the gait component practice capabilities of the FES-IM and gait robot alone and the two combined. During stance phase (Table 4), the gait robot alone provided passive assistance for all gait components, except for lateral weight shift. Lateral weight shift was restricted by the gait robot. The FES-IM system alone induced muscle contraction of the gluteus medius, which assisted pelvic position control in the coronal plane. For the remaining stance-phase pelvic gait components, FES-IM alone did not restrict pelvic movement (that is, FES-IM “allowed” performance, neither restricting nor assisting), but did not assist pelvic gait component movements. That is, the FES-IM system did not restrict any pelvic movements in the transverse plane or the sagittal plane, which are performed in a normal gait pattern (in contrast to the gait robot that restricted all pelvic movements). But the FES-IM system did not facilitate pelvic movement in the transverse or sagittal planes. For the knee and ankle stance-phase gait components, FES-IM alone induced muscle contractions.

During swing phase (Table 4), the gait robot restricted pelvic gait component practice and hip rotation practice. The gait robot alone provided passive assistance for knee and ankle swing-phase gait components and for hip flexion in the sagittal plane. FES-IM alone allowed pelvic movement practice and hip-flexion practice. FESIM alone induced muscle contractions for practice of knee and ankle swing-phase gait components (Table 4). 
Table 4.

Comparison of capability of gait robot alone and functional electrical stimulation with intramuscular electrodes (FES-IM) and combined to deliver practice characteristics of coordinated movement components of stance and swing phases of gait.

\begin{tabular}{|c|c|c|c|}
\hline Gait Component Practice & Gait Robot & FES-IM & Combined \\
\hline \multicolumn{4}{|l|}{ Stance Phase } \\
\hline Forward Weight Shift & Yes, potentially passive & Allowed & Yes, potentially passive \\
\hline Pelvic Position in Coronal Plane & Yes, passively fixed & Yes, muscle activation & Yes, passively fixed \\
\hline Hip Flexion at Initial Contact & Yes, potentially passive & Allowed & Yes, potentially passive \\
\hline Hip Neutral at Midstance & Yes, potentially passive & Allowed & Yes, potentially passive \\
\hline Hip Rotation in Coronal Plane & No, abnormally fixed & Allowed & No, abnormally fixed \\
\hline Knee in Neutral at Initial Contact & Yes, potentially passive & Yes, muscle activation & Yes \\
\hline $\begin{array}{l}\text { Knee in Neutral at Midstance Moving to } 15^{\circ} \\
\text { of Knee Flexion Before Terminal Stance }\end{array}$ & Yes, potentially passive & Yes, muscle activation & Yes \\
\hline Terminal Stance Knee Control & Yes, potentially passive & Yes, muscle activation & Yes \\
\hline Toe Position & Allowed & Yes, muscle activation & Yes \\
\hline \multicolumn{4}{|l|}{ Swing Phase } \\
\hline Pelvic Position Coronal Plane & No, abnormally fixed & Allowed & No, abnormally fixed \\
\hline Pelvic Position Sagittal Plane & No, abnormally fixed & Allowed & No, abnormally fixed \\
\hline Pelvic Rotation & No, abnormally fixed & Allowed & No, abnormally fixed \\
\hline Hip Flexion (Sagittal Plane) & Yes, potentially passive & Allowed & Yes, potentially passive \\
\hline Hip Rotation (Coronal Plane) & No, abnormally fixed & Allowed & No, abnormally fixed \\
\hline Initial Swing Knee Flexion & Yes, potentially passive & Yes, muscle activation & Yes \\
\hline Step Repetition & $\begin{array}{l}40,000 \text { steps over } \\
48 \text { visits }\end{array}$ & $\begin{array}{l}\text { 12,000 steps over } 48 \\
\text { treatment visits }\end{array}$ & $\begin{array}{l}52,000 \text { steps over } \\
48 \text { visits }\end{array}$ \\
\hline $\begin{array}{l}\text { Early Training Capability of Close-to- } \\
\text { Normal Movements }\end{array}$ & $\begin{array}{l}\text { Yes, for all subjects, } \\
\text { passive }\end{array}$ & $\begin{array}{l}\text { Yes, for some subjects, } \\
\text { active }\end{array}$ & Yes, within $1-2$ sessions \\
\hline Reproducibility of Practice Pattern & High & $\begin{array}{l}\text { Variable, depended on } \\
\text { subject ability from session } \\
\text { to session }\end{array}$ & High \\
\hline
\end{tabular}

Table 4 also shows that the gait robot alone provided more step repetition than did FES-IM alone in the protocol provided. A second advantage of the gait robot was that it provided close-to-normal gait component practice early in the protocol, whereas FES-IM alone provided close-to-nor- mal movement for some subjects only later in the protocol, after recovery of some muscle function. The advantage of the FES-IM system was that active muscle contraction was the mode of movement practice. Figure 2 shows a study participant using combined FES-IM and gait robot systems. 


\section{DISCUSSION}

This study extended the literature by demonstrating the feasibility of combining the gait robot and the multichannel FES-IM systems for gait training for subjects in the chronic phase after stroke. The interface of the two systems produced no instances of compromised skin integrity at lead wire exit sites. Subjects perceived the use of the two systems in combination as comfortable. We were able to maintain a clear work space for gait component practice. The clinician was able to effectively use the FES-IM stimulator, finger switch, and external cables during combined gait training with the FES and robotics systems. Clinicians were able to combine interventions after three clinician-training sessions. Both stance- and swingphase FES-IM patterns were used with the gait robot.

We found mixed results regarding the capability of FES-IM and the gait robot systems to provide gait practice characteristics. First, each system afforded unique practice advantages and unique disadvantages. FES-IM alone induced practice of stance-phase hip and knee control through muscle activation of hip abductors and knee flexors and extensors. FES-IM alone also induced practice of swing-phase knee and ankle joint movements and positional control, assisted by or driven by electrically induced muscle contractions. This FES-IM feature might counteract some potentially abnormal muscle activations reported by others for nondisabled controls using the gait robot versus treadmill walking [19]. FES-IM did not restrict any of the coordinated movements of the gait pattern. A disadvantage of gait training with FES-IM alone was that, for some participants, FES-IM was not capable, early in the gait training, of producing a swing-phase knee-flexion movement excursion that was close to normal.

The gait robot alone provided passive or assisted stance-phase hip and knee positioning. For swing phase, the gait robot provided passive or mechanically assisted hip and knee flexion in the sagittal plane. One advantage of the gait robot was that practice of these gait components could occur early in the gait training protocol, before muscle strengthening.

Disadvantages of the gait robot included restriction of lateral weight shift, restriction of pelvic movement to a stationary neutral position, and control of the ankle through a spring-loaded dorsiflexion mechanism that induced a nonregulated ankle dorsiflexion during swing

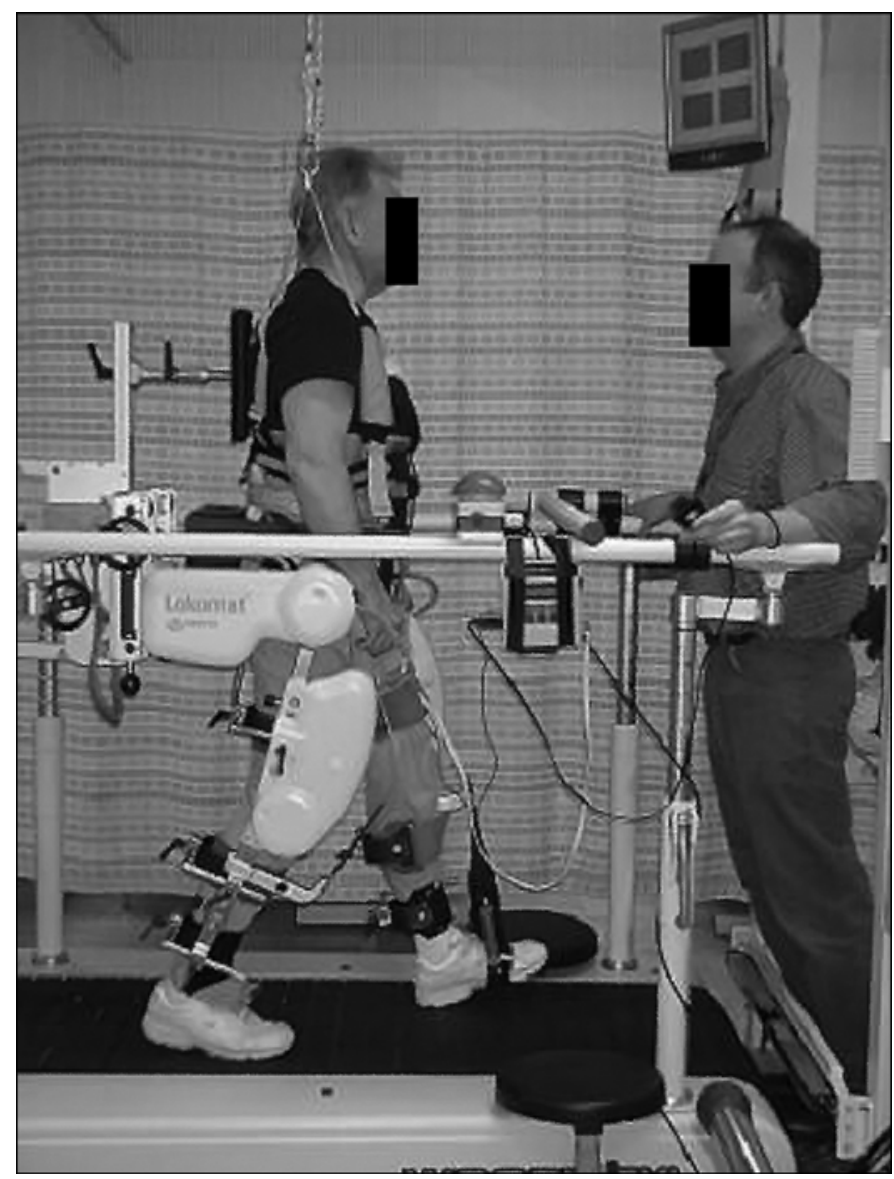

Figure 2.

Study participant receiving combined gait robot and functional electrical stimulation with intramuscular electrodes gait training.

phase. The movement restrictions are important to note for a number of reasons. First, normal walking includes the management of balance control during a lateral weight shift from one limb to the other while simultaneously advancing the center of mass forward over the new stance limb. According to specificity of training principles, if balance control during lateral weight shift is impaired (as it often is after stroke), this complex balance control maneuver of lateral and forward weight transfer must be specifically retrained to be recovered [20-21]. Second, normal gait includes executing pelvic movements that contribute to normal stride length and minimizing the vertical displacement of the center of mass (pelvic rotation in the coronal plane and transverse plane) [22]. If impaired, these pelvic gait components also would need to be specifically retrained. One solution to these two gait robot problems is to retrain lateral weight 
shift and pelvic movements using other methods (e.g., treadmill or overground gait training). Third, a potentially more significant difficulty is that restriction of both lateral weight shift and pelvic movements actually imposes abnormal absence of pelvic movement and abnormal gait practice in this regard.

This study contributed to the literature by providing a description of the capability of the gait robot and FES-IM combined to provide practice of some gait components in a manner that was closer to normal than was otherwise possible. For example, swing-phase knee-flexion GF from the gait robot provided a close-to-normal movement excursion, while FES-IM simultaneously provided muscle activation of knee joint muscle flexors so that the movement was at least partially actively performed, in that muscles were activated. The same combined effect was possible for stance-phase knee and ankle control.

For some practice characteristics, combining the two technologies restricted performance of gait components that would have otherwise been available with the use of one technology alone. For example, the gait robot system restricted pelvic movement, which was allowed (not restricted) by the FES-IM system.

Advantages found in this study regarding the gait robot for stroke gait training were similar to those for spinal cord injury (SCI) gait training. Colombo et al. found that patients with SCI could be provided automated training earlier after trauma, since the gait robot could provide walking assistance forces that were greater than the assistance that physical therapists could provide [23]. They reported that longer duration of gait training could be provided. This study result was consistent with our results of a slightly different nature that showed more steps for a given number of sessions could be practiced with the gait robot or combined FES-IM and gait robot than with only FES-IM. For patients with SCI, the gait robot provided a more reproducible gait pattern from session to session compared with conventional BWSTT [23]. This study result was consistent with the results of the current study. Another advantage of the gait robot for SCI gait training was that only one therapist was needed for training, whereas more than one therapist was often needed to effectively provide BWSTT, especially early in rehabilitation. This finding was consistent with the results of the current study, in that only one therapist was needed for providing combined FES-IM and gait robot training.

\section{CONCLUSIONS}

Combination of multichannel FES-IM and a gait robot was a feasible gait training method. The two technologies combined afforded some advantages. Each technology alone provided unique gait practice characteristics, advantages, and unique disadvantages. Through electrically induced muscle contractions, FES-IM provided subjects practice of swing phase and coordinated limb movements and stance-phase hip, knee, and ankle balance control. The gait robot provided early training, passively assisted, swing-phase hip and knee movements and stance-phase hip, knee, and ankle positional control, which all were very close to normal in joint-movement excursion. For some subjects, FES-IM produced practice of normal excursion of knee and ankle movements during swing phase, which was later in the training, after muscles were sufficiently strengthened to respond to the FES-IM. The gait robot restricted lateral weight shift and pelvic movements, inducing abnormal gait components in that regard. Because of the unique advantages and disadvantages of each alone and the two combined, gait deficits need to be accurately identified and a judicious treatment plan properly targeted before FES-IM, a gait robot, or both combined are selected.

\section{ACKNOWLEDGMENTS}

This material was based on work supported by the VA, Office of Rehabilitation Research and Development, grant B3046I, awarded to Dr. Janis Daly, and Research Career Science award B5080S.

The authors have declared that no competing interests exist.

\section{REFERENCES}

1. Daly JJ, Barnickle K, Kobetic R, Marsolais EB. Electrically induced gait changes post stroke, using an FNS system with intramuscular electrodes and multiple channels. J Neurol Rehabil. 1993;7:17-25.

2. Daly JJ, Ruff RL. Electrically induced recovery of gait components for older patients with chronic stroke. Am J Phys Med Rehabil. 2000;79(4):349-60. [PMID: 10892621]

3. Daly JJ, Ruff RL. Feasibility of combining multi-channel functional neuromuscular stimulation with weight-supported 
treadmill training. J Neurol Sci. 2004;255(1-2):105-15. [PMID: 15465093]

4. Daly JJ, Roenigk KL, Butler KM, Gansen JL, Fredrickson E, Marsolais EB, Rogers J, Ruff RL. Response of sagittal plane gait kinematics to weight-supported treadmill training and functional neuromuscular stimulation following stroke. J Rehabil Res Dev. 2004;41(6A):807-20.

[PMID: 15685469]

5. Daly JJ, Roenigk K, Rogers J, Butler K, Gansen J, McCabe JP. Response of chronic stroke subjects to FNS and weight supported gait training. J Neurol Phys Ther. 2004;28(4):175.

6. Daly JJ, Roenigk K, Holcomb J, Rogers JM, Butler K, Gansen J, McCabe J, Fredrickson E, Marsolais EB, Ruff RL. A randomized controlled trial of functional neuromuscular stimulation in chronic stroke subjects. Stroke. 2006; 37(1):172-78. [PMID: 16322492]

7. O’Sullivan SB, Schmitz TJ. Physical rehabilitation. 5th ed. Philadelphia (PA): F.A. Davis Company; 2007.

8. Visintin M, Barbeau H. The effects of parallel bars, body weight support and speed on the modulation of the locomotor pattern of spastic paretic gait. A preliminary communication. Paraplegia. 1994;32(8):540-53. [PMID: 7970859]

9. Hesse S, Helm B, Krajnik J, Gregoric M, Mauritz KH. Treadmill training with partial body weight support: Influence of body weight release on the gait of hemiparetic patients. J Neurol Rehabil. 1997;11(1):15-20.

10. Trueblood PR. Partial body weight treadmill training in persons with chronic stroke. NeuroRehabilitation. 2001; 16(3):141-53. [PMID: 11790899]

11. Husemann B, Müller F, Krewer C, Heller S, Koenig E. Effects of locomotion training with assistance of a robotdriven gait orthosis in hemiparetic patients after stroke: a randomized controlled pilot study. Stroke. 2007;38(2):349-54. [PMID: 17204680]

12. Jezernik S, Colombo G, Keller T, Frueh H, Morari M. Robotic orthosis Lokomat: A rehabilitation and research tool. Neuromodulation. 2003;6(2):108-15.

13. Kendall FP, EK McCreary, Provance PG, Rogers MM, Romani WA. Muscles: Testing and function with posture and pain. Baltimore (MD): Williams \& Wilkins; 2005.
14. Scheiner A, Polando G, Marsolais EB. Design and clinical application of double helix electrode for functional electrical stimulation. IEEE Trans Biomed Eng. 1994;41(5):425-31. [PMID: 8070801]

15. Marsolais EB, Kobetic R. Implantation techniques and experience with percutaneous intramuscular electrodes in the lower extremities. J Rehabil Res Dev. 1986;23(3):1-8. [PMID: 3490566]

16. Daly JJ, Kollar K, Debogorski AA, Strasshofer B, Marsolais EB, Scheiner A, Snyder S, Ruff RL. Performance of an intramuscular electrode during functional neuromuscular stimulation for gait training post stroke. J Rehabil Res Dev. 2001;38(5):513-26. [PMID: 11732829]

17. Borges G, Ferguson K, Kobetic R. Development and operation of portable and laboratory electrical stimulation systems for walking in paraplegic subjects. IEEE Trans Biomed Eng. 1989;36(7):798-801. [PMID: 2787287]

18. Daly JJ, Nethery J, McCabe JP, Rogers J, Brenner I, Gansen J. Gait Assessment and Intervention Tool (G.A.I.T.). For measurement of coordinated gait components. Physiotherapy. 2007;93 Suppl 1:356.

19. Hidler JM, Wall AE. Alterations in muscle activation patterns during robotic-assisted walking. Clin Biomech (Bristol, Avon). 2005;20(2):184-93. [PMID: 15621324]

20. Plautz EJ, Milliken GW, Nudo RJ. Effects of repetitive motor training on movement representations in adult squirrel monkeys: role of use versus learning. Neurobiol Learn Mem. 2000;74(1):27-55. [PMID: 10873519]

21. Shumway-Cook A, Woollacott MH. Motor control: Translating research into clinical practice. 3rd ed. Philadelphia (PA): Lippincott Williams \& Wilkins; 2007.

22. Neumann DA. Kinesiology of the musculoskeletal system: Foundations for physical rehabilitation. St. Louis (MO): Mosby; 2002.

23. Colombo G, Joerg M, Schreier R, Dietz V. Treadmill training of paraplegic patients using a robotic orthosis. J Rehabil Res Dev. 2000;37(6):693-700. [PMID: 11321005]

Submitted for publication August 16, 2007. Accepted in revised form May 14, 2008. 